MATEC Web of Conferences 23,01024 (2015)

DOI: $10.1051 /$ matec conf/ 20152301024

(C) Owned by the authors, published by EDP Sciences, 2015

\title{
ANALYTICAL APPROACH TO PREDICT THE DRAFT INTENSITY IN THE PREMISES WITH THE HEAT PRODUCING FACILITIES
}

Nikolai I. Kurilenko, Larisa Yu. Mikhailova, Pavel A. Artamonov ${ }^{\mathrm{a}}$

Tyumen State University of Architecture and Civil Engineering, 625001 Tyumen, Russia

\begin{abstract}
The article presents the results of the theoretical and experimental research in the field of the ventilation operating modes of the unit-type heat producing facilities. The analytical dependence is given to estimate the draft intensity indoors of the boiler room. The test data are compared to the derived analytical dependences.
\end{abstract}

\section{Introduction}

One of the characteristic features of the ventilation systems of the premises with the heat producing facilities is that a large volume of the air, intended for combustion and general ventilation, inflows through the vents into the comparatively small rooms. Due to the very compact arrangement of the pipes and small size of the premises, the great excess of the sensible heat is observed in the premises with the heat producing facilities. In addition, the assembly technology of the sandwich-type enclosing structures ensures the construction of the premises of the high degree air-tightness. These factors cause the requirement of the special approach to consideration of the air regime of this type of the premises.

\section{Problem statement and solution methods}

The heat and mass transfer processes in the premises with the heat producing facilities can be considered as an enclosed volume with the inner heat release, inlet and outlet, see Fig. 1.

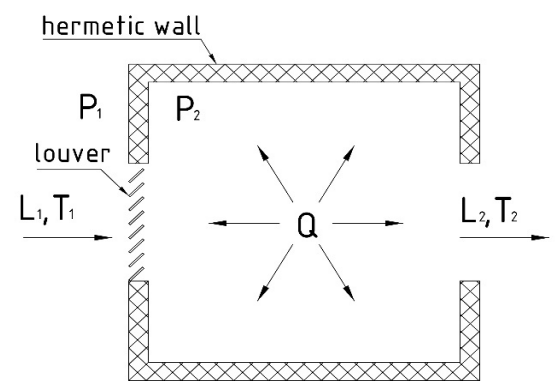

Figure 1. The analytical model of the premises with the heat producing facilities

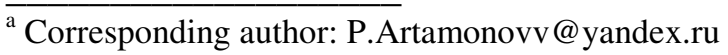


Then the value of the pressure drop across the pressure $\mathrm{P}_{2}$ inside the volume under consideration and the pressure of the medium of the inflowing air $\mathrm{P}_{1}$ can be calculated by Darcy-Weisbach formula [1]:

$$
\Delta P=\sum \xi_{\text {пр }} \frac{v^{2} \rho_{\text {н.в. }}}{2}
$$

where $\Delta P$ - the draft in the premises with the heat producing facilities, $\mathrm{Pa}, \Sigma \xi_{\text {пр }}$ - the sum of the local resistance coefficients on the air inlets (louvers, draft-proof non-return valves etc.), $\rho_{\text {н.в. }}-$ the density of the outdoor air, $\mathrm{kg} / \mathrm{m}^{3}, v-$ the air velocity through the louver, $\mathrm{m} / \mathrm{s}$. According to [2] the velocity is calculated as follows (2):

$$
v=\frac{L_{1}}{F}
$$

where $F$ - the discharge area of the inlet, $\mathrm{m}^{2}, L_{1}$ - the air-flow rate for combustion, $\mathrm{m}^{3} / \mathrm{s}$, in case if $L_{1}=L_{2}$ is calculated by the formula (3):

$$
L_{1}=L_{2}=B \cdot V_{\mathrm{o}} \cdot \alpha
$$

where $\alpha$ - an excess air ratio, $V_{\mathrm{o}}$ - the theoretically required air volume for the fuel combustion, $\mathrm{m}^{3} / \mathrm{nm}^{3}\left(\mathrm{~m}^{3} / \mathrm{kg}\right), B-$ the fuel consumption, $\mathrm{m}^{3} / \mathrm{s}(\mathrm{kg} / \mathrm{s})$, which according to [3], can be calculated by the formula (4):

$$
B=\frac{Q_{\mathrm{K}}}{Q_{\mathrm{H}}^{\mathrm{p}} \cdot \eta}
$$

where $Q_{\mathrm{K}}$ - the heating capacity of the heat producing facilities, $\mathrm{kW}, Q_{\mathrm{H}}{ }^{\mathrm{p}}-$ the low heating value of the workable fuel composition, $\mathrm{kJ} / \mathrm{nm}^{3}(\mathrm{~kJ} / \mathrm{kg}), \eta$ - the boiler efficiency.

The heat production on the state-of-the-industry heat producing facilities is a dynamic process. This means that the fuel consumption and, consequently, the air-flow rate through the supply louver is not constant. To account for the instable air-flow rate in the volume under review, we introduce the coefficient «a», which shows the percent of the used heat power of the facility. Then, in view of the equation (4) and the coefficient «a», the air-flow rate for the combustion can be recorded by the following formula (5):

$$
L_{2}=\frac{a \cdot Q_{\mathrm{K}} \cdot \alpha \cdot V_{\mathrm{o}}}{Q_{\mathrm{H}}^{\mathrm{p}} \cdot \eta}
$$

The air - flow rate, calculated by the formula (5), can be used to define the pressure drop subject to the formula (1) only if the flow of the supply air is isothermal, i.e. $\mathrm{L}_{1}=\mathrm{L}_{2}$ (see Fig. 1). This statement is not valid for the type of the premises under review, because there is an excess heat $\mathrm{Q}$, that add in the inflowing air heating.

The real values of the operation pressure and temperature of the objects under review allow using the ideal gas model to describe the physical processes [4]. On the supposition that the difference between $\mathrm{P}_{1}$ and $\mathrm{P}_{2}$ is so small that it is acceptable to take $\mathrm{P}_{1}=\mathrm{P}_{2}$ for the practical purposes. Then the $\mathrm{L}_{1} / \mathrm{L}_{2}$ ratio can be written as an expression (6), which is a consequence of the Charles's law [5]:

$$
\frac{L_{1}}{L_{2}}=\frac{T_{1}}{T_{2}}
$$

At that the flow through the supply louver with allowance for non-isothermal flow subject to the ideal gas law with an error equal to $\mathrm{P}_{1} / \mathrm{P}_{2}$ can be found by the formula (7):

$$
L_{1}=\frac{T_{1}}{T_{2}} \cdot L_{2}=\frac{T_{1}}{T_{2}} \cdot\left(\frac{a \cdot Q_{\mathrm{K}} \cdot \alpha \cdot V_{\mathrm{o}}}{Q_{\mathrm{H}}^{\mathrm{p}} \cdot \eta}\right)
$$

Using the equations (1), (2) and (7) the draft intensity under non-isothermal flow in the premises with the heat producing facilities one can derive the following equation (8): 


$$
\Delta P=\frac{\sum \xi_{n p} \rho_{\text {н.в. }}}{2} \cdot\left(\frac{T_{1}}{T_{2} \cdot F} \cdot \frac{a \cdot Q_{\mathrm{K}} \cdot \alpha \cdot V_{\mathrm{o}}}{Q_{\mathrm{H}}^{\mathrm{p}} \cdot \eta}\right)^{2}
$$

An experiment was performed to compare the real values of the draft intensity and the one calculated by the formula (8).

The experiment was performed in the premises with two installed fire-tube heat generators «KSB0,5» brand powered by natural gas. There used two-phase supercharged burners by «CibUnigas» production. The air intake was through the louvers under negative pressure created by the burners. The measurements were performed with two instruments. The first instrument - electronic differential pressure gauge «Wohler DM 2000» graduated in $1 \mathrm{~Pa}$. The second - differential pressure gauge «Testo $480 »$ graduated in $0,1 \mathrm{~Pa}$. The outdoor air temperature during the measurements was $5^{\circ} \mathrm{C}$ below zero. The measurements were performed under calm weather. The instrument was mounted on the inner surface of the wall, thus one of the nipples measured the internal air pressure and the second nipple of the differential pressure gauge measured the air pressure outside the room with the pulse tube. Every instrument did 20 measurements of the pressure difference. The measurements were done at 5 second interval. The total value is calculated by averaging out of 20 values under every measurement mode. To fix the draft intensity created by the burner only, the general ventilation was shut off and the vent of the induced draught fan was sealed for the period of measurements. The doorways and other locations of the possible air inflow were not sealed. The measurements were done under six modes of the boiler operation, see Table 1 . In order to derive the draft intensity created by the burner only, the differential pressure value was measured with the burner-off, see modes 3,6 .

Table 1

Operating modes of the boiler room

\begin{tabular}{|c|c|c|}
\hline Mode & Number of the open supply louvers & Operating mode of the burner \\
\hline 1 & 2 & Min \\
\hline 2 & 2 & Max \\
\hline 3 & 2 & Off \\
\hline 4 & 1 & Min \\
\hline 5 & 1 & Max \\
\hline 6 & 1 & Off \\
\hline
\end{tabular}

In order to use the formula (8), during the experiment the measurements were done for the real parameter values of the heat generator operation. The air temperature in the room was $29^{\circ} \mathrm{C}$ above zero. The readings of the gas-analyzer were used to calculate the real value of the boiler efficiency by equation 1 [6], wherein the total losses in the environment and incomplete combustion were taken as $0,45 \%$. The parameters of the gas flow rate were read on the turbine flow meter of the boiler gas pipeline: for the maximum load the gas flow rate makes up $60 \mathrm{~m}^{3} /$ hour, for the minimal mode of the burner $-3,5 \mathrm{~m}^{3}$ /hour. The calculated component composition of the natural gas at n. f. y.: $\mathrm{CH}_{4}-96 \%$, $\mathrm{C}_{2} \mathrm{H}_{6}-1,65 \%, \mathrm{C}_{3} \mathrm{H}_{8}-0,6 \%, \mathrm{C}_{4} \mathrm{H}_{10}-0,23 \%, \mathrm{~N}_{2}-1,41 \%, \mathrm{CO}_{2}-0,11 \%$. The low heat value of the gas $-36349 \mathrm{~kJ} / \mathrm{m}^{3}$. The theoretical amount of air required for combustion of one cubic meter of the gas, calculated on account of the gas composition $-10,81 \mathrm{~nm}^{3} / \mathrm{nm}^{3}$. The results of calculations by the formula (8) are given in Table 3, lines \# 1, 3.

Also the computing simulation was done for analysis of the resultant dependency. The volume with the real geometry of the supply louvers constructed by size of the premises with the heat producing facilities, where the experiment performed, was used as a domain of computation. The process simulation was based on the finite element method [7], [8], [9]. Here are the boundary conditions accepted in the model: the burner performance was set depending on the heat capacity, the indoor and outdoor atmospheric pressure and the room temperature of $268 \mathrm{~K}$ were taken as the initial conditions, the level of the non-isothermal flow was caused by the set value of the heat flux off the heat generator surface. The enclosures were taken as completely airtight. During the simulation the continuity equation was solved (9): 


$$
\frac{\partial \rho}{\partial t}+\nabla \bullet(U \rho)=0
$$

where $\rho$ - the density of the medium, $\mathrm{kg} / \mathrm{m}^{3} ; t$ - time, $\mathrm{c} ; U$ - the velocity vector, $\mathrm{m} / \mathrm{s}$; The impulse law is described by the equation (10):

$$
\frac{\partial(U \rho)}{\partial t}+\nabla \bullet(U \rho \otimes U)=-\nabla p+\nabla \bullet \tau+S_{M}
$$

where $p$ - the absolute pressure, $\mathrm{Pa} ; S_{M}$ - the mass volume source, $\mathrm{kg} / \mathrm{m}^{2} \cdot \mathrm{c}^{3} ; \tau$ - the stress tensor is described by the equation (11):

$$
\tau=\mu\left(\nabla U+(\nabla U)^{T}-\frac{2}{3} \delta \nabla \bullet U\right)
$$

where $\mu$ - the dynamic viscosity, $\mathrm{Pa} \cdot \mathrm{s} ; \delta$ - the Kronecker function; $\mathrm{T}$ - temperature, $\mathrm{K}$;

The energy change was calculated subject to the following equation (12):

$$
\frac{\partial(\rho e)}{\partial t}+\nabla \bullet(\rho U e)=\nabla \bullet(\lambda T)+p \nabla \bullet U+\tau: \nabla U+S_{E}
$$

where $e$ - the internal energy; $\tau: \nabla U$ - viscous dissipation; $\mathrm{S}_{\mathrm{E}}-$ the energy volume source, $\mathrm{kg} / \mathrm{m} \cdot \mathrm{s}^{3}$.

The results obtained during the computer simulation are presented in Table 3, lines \# 2, 4.

\section{Results and discussion}

\begin{tabular}{|c|c|c|c|c|c|c|c|c|c|c|c|c|}
\hline \multirow[b]{2}{*}{ Measurement \# } & \multicolumn{6}{|c|}{$\begin{array}{l}\text { The draft measured with «Wohler } \\
\text { DM 2000», Pa }\end{array}$} & \multicolumn{6}{|c|}{$\begin{array}{l}\text { The draft measured with «Testo } \\
480 », \mathrm{~Pa}\end{array}$} \\
\hline & 1 & 2 & 3 & 4 & 5 & 6 & 1 & 2 & 3 & 4 & 5 & 6 \\
\hline 1 & 5 & 5 & 2 & 6 & 11 & 1 & 2.6 & 4 & 0.4 & 5.2 & 9.3 & 1.2 \\
\hline 2 & 5 & 6 & 2 & 7 & 9 & 2 & 2.5 & 4 & 0.3 & 5.2 & 9.1 & 1.2 \\
\hline 3 & 4 & 4 & 1 & 6 & 8 & 2 & 2.4 & 3.3 & 0.4 & 5.2 & 8.9 & 1.2 \\
\hline 4 & 3 & 6 & 1 & 5 & 8 & 2 & 2.1 & 3.3 & 0.4 & 5.2 & 8.9 & 1.2 \\
\hline 5 & 2 & 7 & 1 & 5 & 8 & 1 & 1.9 & 3.1 & 0.5 & 5.6 & 8.9 & 1 \\
\hline 6 & 4 & 7 & 2 & 5 & 10 & 1 & 1.9 & 3 & 0.5 & 5.7 & 9 & 1 \\
\hline 7 & 2 & 5 & 1 & 6 & 9 & 1 & 1.6 & 3.6 & 0.5 & 5.6 & 9.1 & 1 \\
\hline 8 & 2 & 6 & 1 & 6 & 10 & 2 & 1.6 & 3.6 & 0.5 & 5.8 & 9.3 & 0.9 \\
\hline 9 & 3 & 4 & 1 & 4 & 8 & 1 & 1.7 & 3.8 & 0.6 & 5.9 & 9.4 & 0.6 \\
\hline 10 & 4 & 6 & 3 & 5 & 9 & 1 & 1.9 & 3.8 & 0.7 & 5.7 & 8.7 & 0.5 \\
\hline 11 & 3 & 7 & 1 & 6 & 9 & 1 & 2.5 & 4.1 & 0.8 & 5.5 & 8.9 & 0.4 \\
\hline 12 & 3 & 5 & 2 & 6 & 10 & 1 & 2 & 4.1 & 1 & 5.3 & 8.9 & 0.6 \\
\hline 13 & 4 & 6 & 2 & 4 & 10 & 2 & 2 & 4.2 & 0.8 & 5 & 8.8 & 1 \\
\hline
\end{tabular}

The test measurements results are given in Table 2.

The test measurement results

Table 2 


\begin{tabular}{|c|c|c|c|c|c|c|c|c|c|c|c|c|}
\hline 14 & 3 & 6 & 1 & 4 & 8 & 2 & 2.5 & 4 & 0.6 & 5.3 & 9.7 & 1.3 \\
\hline 15 & 4 & 7 & 1 & 5 & 10 & 2 & 2.2 & 3.8 & 0.5 & 5.3 & 9.7 & 1.4 \\
\hline 16 & 3 & 5 & 1 & 6 & 8 & 1 & 2.2 & 4 & 0.5 & 5.4 & 9.5 & 1.4 \\
\hline 17 & 4 & 5 & 1 & 6 & 8 & 1 & 2.3 & 4 & 0.6 & 5.6 & 9.4 & 1.4 \\
\hline 18 & 5 & 4 & 1 & 7 & 9 & 2 & 2.3 & 3.9 & 0.6 & 6 & 9.3 & 1.3 \\
\hline 19 & 5 & 5 & 2 & 5 & 9 & 1 & 2.5 & 4 & 0.8 & 6.2 & 9 & 1.4 \\
\hline 20 & 4 & 6 & 1 & 4 & 9 & 1 & 2.6 & 3.6 & 0.7 & 5.7 & 8.9 & 1.4 \\
\hline Average value & 3.6 & 5.6 & 1.4 & 5.4 & 9 & 1.4 & 2.2 & 3.8 & 0.6 & 5.5 & 9.1 & 1.1 \\
\hline $\begin{array}{l}\text { The draft created by the } \\
\text { burner }\end{array}$ & 2.2 & 4.2 & & 4 & 7.6 & & 1.6 & 3.2 & & 4.5 & 8.1 & \\
\hline
\end{tabular}

The draft intensity created in the burner under modes 1 and 2 is derived as the difference between the average measured value under modes 1 and 2 and the average value of mode 3 . For modes 4 and 5 the draft intensity is derived as the difference of the average measured values under modes 4 and 5 and the average value of mode 6 .

The results of the theoretical and empirical values of the draft intensity are presented in Table 3 .

Table 3

The draft intensity in the premises with the heat producing facilities

\begin{tabular}{|c|c|c|c|c|c|c|c|}
\hline & & & H & & & & \\
\hline & & & & 1 & 2 & 4 & 5 \\
\hline$\dot{\mathscr{D}} \dot{\mathscr{\theta}}$ & 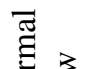 & Calculated by the formula (8) & 1 & 1,394 & 5,387 & 5,428 & 21,051 \\
\hline 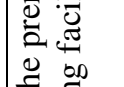 & 蕓 & $\begin{array}{l}\text { Defined by the results of the } \\
\text { computer simulation }\end{array}$ & 2 & 1,310 & 5,011 & 5,575 & 21,549 \\
\hline$\Xi \stackrel{\bar{v}}{\bar{z}}$ & 3 & Calculated by the formula (8) & 3 & 1,098 & 4,243 & 4,391 & 16,972 \\
\hline 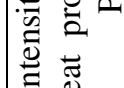 & $\overline{\Xi ี \Xi}$ & $\begin{array}{l}\text { Defined by the results of the } \\
\text { computer simulation }\end{array}$ & 4 & 1,068 & 4,098 & 4,428 & 16,730 \\
\hline 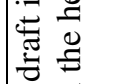 & $\begin{array}{l}\frac{\pi}{0} \\
. \frac{2}{1}\end{array}$ & $\begin{array}{l}\text { The value measured with the } \\
\text { instrument Wohler } 2000\end{array}$ & 5 & 2,200 & 4,200 & 4,000 & 7,600 \\
\hline$\stackrel{8}{E}$ & z̃ & $\begin{array}{l}\text { The value measured with the } \\
\text { instrument Testo } 480\end{array}$ & 6 & 1,580 & 3,180 & 4,450 & 8,070 \\
\hline
\end{tabular}

\section{Conclusions}

Table 3 allows making the following conclusions:

- The solution of equation (8) very accurately matches the results of the computer simulation of the processes of the outside air inflowing the premises with the heat producing facilities under complete integrity of the enclosures.

- At the burner powerup there is a significant deviation of the draft intensity real value from the theoretical value defined by a model with the completely sealed enclosures, see Table 3 , mode 5 . Therefore, the difference between the real value measured with the differential pressure gauge and the 
theoretical value calculated by the formula (8) is the characteristic of the enclosures air permeability of the premises with the heat producing facilities, and, in practice, this makes it possible to evaluate the air permeability of this type premises only upon readings of the differential pressure gauge.

\section{References}

1. P.N. Kamenev, E.I. Tertichnik, Ventilation: a tutorial 94, 616 (2008).

2. V.F. Drozdov, Heating and ventilation. Part II Ventilation. 105, 262 (1984).

3. Thermal design of boiler (Standard method) third edition, revised and enlarged 32, 256 (1998).

4. V.A. Kuzovlev, Technical thermodynamics and heat transfer bases 8, 335 (1983).

5. A.G. Stromberg, D.P. Semchenko, Physical chemistry 87, 480 (1973).

6. N.I. Kurilenko, L.Yu. Mikhailova, P.A. Artamonov, Features air mode block automated boiler installations. Russ. Privolzhsky scientific journal 116, 152 (2014).

7. ANSYS HELP: Chapter 4: Mathematical Models of CFX http://www.iesd.dmu.ac.uk/.

8. A.B. Kaplun, E.M. Morozov, M.A. Olfer'eva. ANSYS in the hands of the engineer: A Practical Guide, 272 (2003).

9. K.A. Basov, ANSYS and LMS Virtual Lab. Geometric modeling, 240 (2006). 